\title{
Searching for gravitational waves from known pulsars
}

\author{
Matthew Pitkin for the LIGO Scientific Collaboration \\ Department of Physics and Astronomy, Kelvin Building, University of Glasgow, \\ Glasgow G12 8QQ, UK \\ E-mail: matthew@astro.gla.ac.uk
}

\begin{abstract}
We present upper limits on the amplitude of gravitational waves from 28 isolated pulsars using data from the second science run of LIGO. The results are also expressed as a constraint on the pulsars' equatorial ellipticities. We discuss a new way of presenting such ellipticity upper limits that takes account of the uncertainties of the pulsar moment of inertia. We also extend our previous method to search for known pulsars in binary systems, of which there are about 80 in the sensitive frequency range of LIGO and GEO 600.
\end{abstract}




\section{Introduction}

Over the last few years the LIGO and GEO 600 gravitational wave detectors have had three (S1, S2 and S3) and two (S1 and S3) science runs respectively. Data from S1 have been used to set upper limits on gravitational wave emission from a variety of possible sources [1, 2, 3, 4]. Here we concentrate on a search for gravitational waves from known pulsars which has been extended both in terms of analysis method and number of sources from that presented in [1].

S1 (23 August - 9 September 2002) was the first major data taking period and a search for gravitational waves from only one pulsar (the fastest millisecond pulsar J1939+2134) was performed [1. This search used both a time domain and a frequency domain analysis method. For S2 (14 February - 14 April 2003) the number of pulsars being searched for increased to 28 [5], with the criterion that the pulsars were isolated and had spin frequencies within the sensitive region of the instruments, i.e. $f>25 \mathrm{~Hz}$ or gravitational wave frequencies of $f_{\mathrm{GW}}>50 \mathrm{~Hz}$, assuming emission from a triaxial neutron star [6]. The time domain analysis technique was considered the optimal search for S2 where the source position and frequency parameters were known. The analysis and results from $\mathrm{S} 2$, including possible astrophysical interpretations, will be summarised in this paper.

Analysis of the S3 run (31 October 2003 - 9 January 2004) is underway and has the aim of extending the number of pulsars in the search to all those with $f>25 \mathrm{~Hz}$ $(\sim 110)$ including those within binary systems. The additions to the analysis needed to incorporate the binary systems will be discussed in more detail in this paper. We will also discuss how the results on neutron star ellipticity could be interpreted as an exclusion region on a moment of inertia - ellipticity plane.

\section{S2 summary}

\subsection{Analysis}

S2 data were available for analysis from all three LIGO interferometers. Frequency and positional information for all the isolated pulsars with $f>25 \mathrm{~Hz}$ can be found on the Australia Telescope National Facility (ATNF) [7] online catalogue. However for some pulsars this information lacked the accuracy required to be sure that the search was coherent over the period of the science run, and additional timing over the epoch of S2 was requested and obtained for $18 \ddagger$ of these objects [8]. In total this gave us 28 pulsars with reliable information upon which to reconstruct their phase evolution. Of these 28 pulsars, 14 are in globular clusters. The list also includes the Crab pulsar (J0534+2200) and the fastest millisecond pulsar J1939+2134 targeted in S1.

The analysis method is described in detail in [9]. In summary we perform a time domain heterodyne of the data with the known phase evolution of the pulsar signal.

‡ The Crab pulsar $(J 0534+2200)$ data was taken from the online Jodrell Bank Monthly ephemeris http://www.jb.man.ac.uk/research/pulsar/crab.html 


\begin{tabular}{c|c}
$h_{0}^{95 \%}$ & no. of pulsars \\
\hline $1 \times 10^{-24}<h_{0} \leq 5 \times 10^{-24}$ & 20 \\
$5 \times 10^{-24}<h_{0} \leq 1 \times 10^{-23}$ & 4 \\
$h_{0}>1 \times 10^{-23}$ & 4 \\
\hline
\end{tabular}

Table 1. The $95 \%$ upper limits on $h_{0}$ for the 28 pulsar searched for with the S2 run.

\begin{tabular}{c|c} 
ellipticity $\epsilon$ & no. of pulsars \\
\hline $1 \times 10^{-6}<\epsilon \leq 1 \times 10^{-5}$ & 4 \\
$1 \times 10^{-5}<\epsilon \leq 1 \times 10^{-4}$ & 16 \\
$\epsilon>1 \times 10^{-4}$ & 8 \\
\hline
\end{tabular}

Table 2. The upper limits on $\epsilon$ for the 28 pulsar searched for with the S2 run.

After heterodyning, any signal in the data would vary only with the beam pattern [6] of the detector and its form would depend on the four unknown parameters of gravitational wave amplitude $\left(h_{0}\right)$ and polarisation angle $(\psi)$, pulsar orientation angle $(\iota)$ and the signal's initial phase $\left(\phi_{0}\right)$. The heterodyned data is then filtered and re-binned from the detector sample rate of $16384 \mathrm{~Hz}$ to $1 / 60 \mathrm{~Hz}$. We then determine the probability distribution functions of the parameter values using a Bayesian inference technique, as shown in 9 .

\subsection{Hardware injections}

During S2 we injected two artificial pulsar signals into the three LIGO interferometers for 12 hours by modulating one mirror of each via the actuation control signal. This was done to perform a validation of the search pipeline from as far up the chain as possible. The injections increased our confidence in the phase calibration of the detectors, and allowed for a joint coherent analysis of data from all the detectors.

\subsection{Results and astrophysical interpretation}

The $95 \%$ upper limits on $h_{0}$ are summarised in Table. [1. We can recast this upper limit on $h_{0}$ as an upper limit on the pulsar's ellipticity, $\epsilon$, calculated via

$$
\epsilon \simeq 0.237 \frac{h_{0}}{10^{-24}} \frac{r}{1 \mathrm{kpc}} \frac{1 \mathrm{~Hz}^{2}}{f^{2}} \frac{10^{38} \mathrm{~kg} \mathrm{~m}^{2}}{I_{z z}}
$$

where $r$ is the distance to the pulsar in kpc, $f$ is its spin frequency, and $I_{z z}$ is its principal moment of inertia [5]. The ellipticity limits are summarised in Table 2 using the canonical value of $10^{38} \mathrm{~kg} \mathrm{~m}^{2}$ for the moment of inertia and assuming no error on the pulsar's distance. The lowest value of strain upper limits $\left(1.7 \times 10^{-24}\right)$ is associated with pulsar J1910-5959D and of ellipticity $\left(4.5 \times 10^{-6}\right)$ with pulsar J2124-3358. The upper limit for the Crab pulsar, at $h_{0}=4.1 \times 10^{-23}$, is only a factor of $\sim 30$ greater than the 
canonical limit inferred from simple spin-down arguments§. For all other pulsars the upper limits are a few orders of magnitude higher than their canonical limits.

Although the inferred ellipticities are well above those allowed by the majority of conventional neutron star equations of state they are approaching the region of astrophysical interest. Indeed, for the lowest pulsar ellipticities $\left(\sim 4.5 \times 10^{-6}\right)$ we are starting to reach the range permitted by at least one exotic theory of neutron star structure [11. It should be stressed that these results are the first direct upper limits on gravitational wave emission for 26 of the 28 pulsars, with previous upper limits for pulsar J1939+2134 and the Crab pulsar given by [1] and [10] respectively. For the pulsars within globular clusters the spin-down can be masked by local Doppler shifts caused by the cluster dynamics. This makes placing a spin-down based upper limit difficult unless the pulsar motions within the clusters can be found independently. These gravitational wave interferometer upper limits are inherently independent of the cluster dynamics.

\section{S3 analysis}

We expanded the search in the S3 analysis to include all pulsars with $f>25 \mathrm{~Hz}$. These include $\sim 70$ pulsars within binary (or more complex) systems so it is important to account for the extra Doppler and relativistic time delays due to the pulsar's orbital motion. An advantage over S2 is the availability of GEO 600 data in addition to LIGO data.

\subsection{Binary pulsar signal}

In the case of an isolated pulsar the signal received at the detector needs to be corrected to the solar system barycentre (SSB) by calculating the Doppler delays and other relativistic effects. This is possible as the pulsar's position is known and we have very good solar system ephemerides. Of course it is simply the reverse procedure to that used to determine the positional and frequency parameters of the pulsar from radio data, so the reconstruction is generally of good quality. The motion of a pulsar in a binary system adds a number of Doppler and relativistic time delays:

$$
\Delta T_{\text {bin }}=\Delta_{\mathrm{R}}+\Delta_{\mathrm{E}}+\Delta_{\mathrm{S}}+\Delta_{\mathrm{A}}
$$

where $\Delta_{R}$ is the Roemer delay (light travel time), $\Delta_{E}$ is the Einstein delay due to special relativistic effects, $\Delta_{\mathrm{S}}$ is the general relativistic Shapiro delay and $\Delta_{\mathrm{A}}$ is the abberation delay caused by the pulsar's rotation. These delay changes can be far more pronounced than those from the Earth's motion, with up to a $0.03 \mathrm{~Hz}$ frequency shift. The time delays are parameterised by properties of the binary system including its period, eccentricity, angular velocity, time of periastron, projected semi-major axis and several relativistic parameters depending on the nature of the system. These parameters are found by fitting the radio observations (using the standard TEMPO data reduction $\S$ This limit is imposed by energy conservation and assumes that the rotational kinetic energy is only lost to gravitational waves 
package [12]) to various binary models (e.g. 13]). The model to which the binary system is fitted will depend on how relativistic it is or which parameters you wish to extract. The 70 binary system pulsars fall mainly into two models: 32 in the low eccentricity (ELL1 model [14]) and 33 in the Blandford-Teukolsky (BT model [13]). Four fall into the highly relativistic Damour-Deruelle (DD [13) model and a further one into the Blanford-Teukolsky-2-Planet (BT2P) model, although this can be adequately fit using the simpler BT model. With this additional binary information provided the full set of pulsars can be included in the search. So far new timing information for the majority of these pulsars has been provided 8 and the analysis is underway.

\subsection{Hardware injections}

We injected ten artificial isolated pulsar signals, with a wide range of signal parameters into the LIGO interferometers. One other signal was injected into both GEO 600 and LIGO. The signal strengths ranged from marginally detectable to very strong. These signals have been successfully extracted using the search pipeline [9]. For the strongest signals, agreement between the injected and recovered parameter values has been to within a few percent.

\section{Moment of inertia - ellipticity plane}

So far we have used the canonical value for the neutron star moment of inertia $\left(10^{38} \mathrm{~kg} \mathrm{~m}^{2}\right)$ when calculating the pulsar ellipticities. This is the moment of inertia of a $1.4 \mathrm{M}_{\odot}$ sphere of uniform density and radius $10 \mathrm{~km}$. The true value can vary by factors of a few under different models for the neutron star equation of state. Equation 1 shows that $h_{0}$ can be used to set an upper limit on the neutron star's quadrupole moment, $I_{z z} \epsilon$, that is independent of the actual value of $I_{z z}$. This value can then be used to form an exclusion region in the $I_{z z}-\epsilon$ plane (see Fig. (1). This plane can provide exclusion regions on both the moment of inertia and ellipticity or can be used to read off an upper limit on ellipticity for a preferred value of $I_{z z}$ such as the canonical value.

Figure 2 shows such an exclusion region for the Crab pulsar upper limit from S2. An exclusion region can also be set using the spin-down based upper limit argument, at

$$
I_{z z} \epsilon=\frac{5|2 \pi \dot{f}| c^{5}}{32 G(2 \pi f)^{5}} \frac{1}{\epsilon} .
$$

The point at which the experimental results and the spin-down result cross shows the the point at which the experimental results beats that of spin-down. For the S2 Crab pulsar result it can be seen that the experimental result only beats the spin-down result for unfeasably large values of $I_{z z}\left(>8 \times 10^{40} \mathrm{~kg} \mathrm{~m}^{2}\right)$. The upper limit on the ellipticity for the experimental result and assuming $I_{z z}=10^{38} \mathrm{~kg} \mathrm{~m}^{2}$ is $\sim 2 \times 10^{-2}$. 


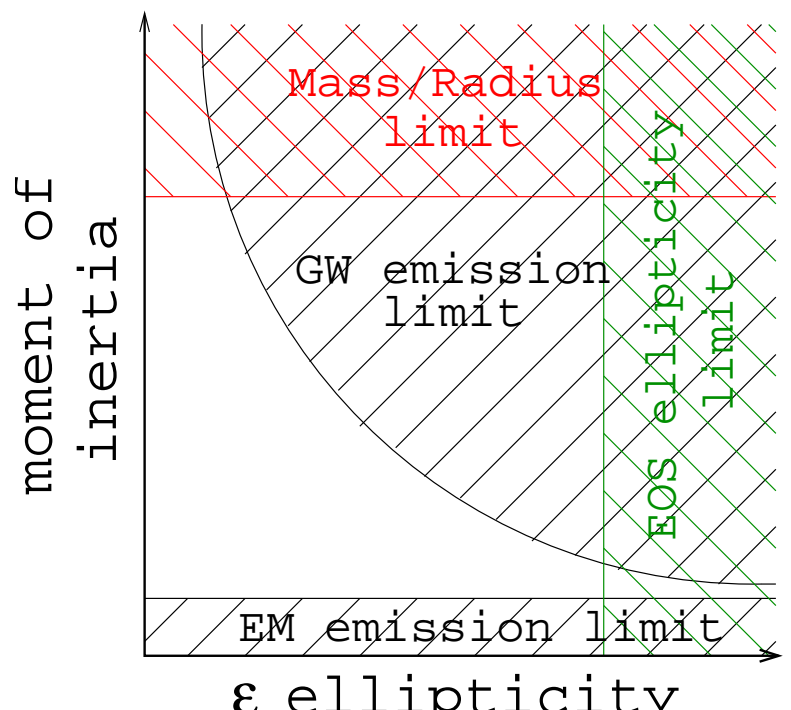

Figure 1. This figure shows a rough representation of the regions in the moment of inertia $I_{z z}$ - ellipticity $\epsilon$ plane that can be excluded via various methods. The electromagnetic emission of a pulsar can set a lower limit on the moment of inertia by equating the EM emission with the rotational energy loss of the pulsar 15. The various equations of state for neutron stars can constrain the mass/radius relation and therefore moment of inertia. Equations of state will also put limits on the maximum allowable ellipticity of the neutron star. A limit can be set from upper limits on gravitational wave emission.

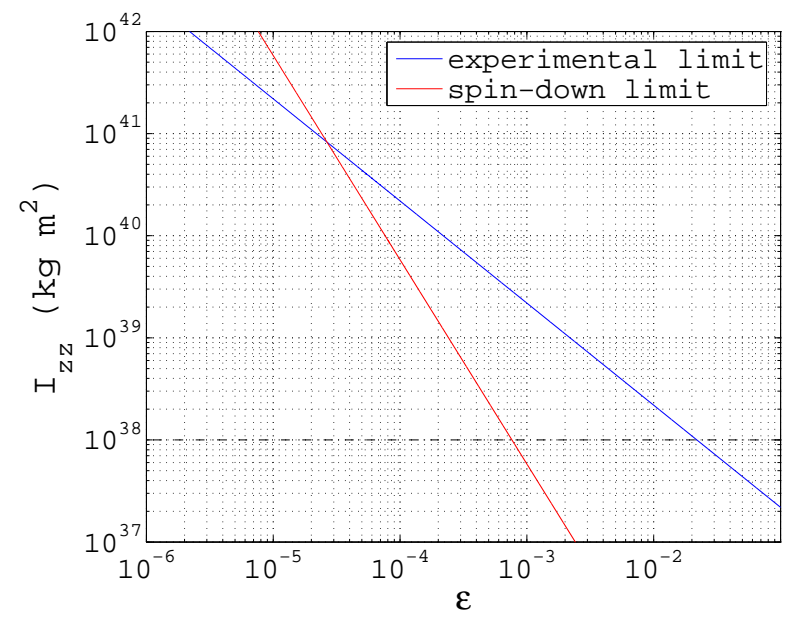

Figure 2. The areas above the full lines are the excluded regions on the moment of inertia - ellipticity plane for the Crab pulsar as obtained from the S2 $h_{0}$ upper limit and the spin-down arguments. These assume a distance to the Crab pulsar of $2 \mathrm{kpc}$. 


\section{Future work}

The analysis of S3 data is presently underway targeting most known pulsars within the band. In addition we will be incorporating other sources of error into the current Bayesian framework, including systematic calibration errors in the value of $h_{0}$ and pulsar distance errors into the value of $\epsilon$ or the $I_{z z}-\epsilon$ plane exclusion region. The S3 results promise to give a reasonable improvement over S2 for some pulsars. In particular, the Crab pulsar result should only be a factor of a few above the spin-down limit.

The next science run of LIGO and GEO 600 (S4) is due to be underway in early 2005 providing more data to analyse. The pipeline used to obtain the results in this paper is at a mature stage and will be applied directly to the S4 data.

\section{References}

[1] Abbott B et al (The LIGO Scientific Collaboration) 2004 Phys. Rev. D 69082004

[2] Abbott B et al (The LIGO Scientific Collaboration) 2004 Phys. Rev. D 69122001

[3] Abbott B et al (The LIGO Scientific Collaboration) 2004 Phys. Rev. D 69102001

[4] Abbott B et al (The LIGO Scientific Collaboration) 2004 Phys. Rev. D 69122004

[5] Abbott B et al (The LIGO Scientific Collaboration), Kramer M and Lyne A, accepted by Phys. Rev. Lett. Preprint gr-qc/0410007

[6] Jaranowski P, Królak A and Schutz B 1998 Phys. Rev. D 58063001

[7] http://www.atnf.csiro.au/research/pulsar/psrcat/

[8] Kramer M and Lyne A, Jodrell Bank, private communication.

[9] Dupuis R J 2004 PhD Thesis, University of Glasgow

[10] Suzuki T 1995, in First Edoardo Amaldi Conference on Gravitational Wave Experiments, edited by E. Coccia, G. Pizzella and F. Ronga (World Scientific, Singapore) p 115

[11] Owen B J, submitted to Phys. Rev. Lett. Preprint astro-ph/0503399

[12] http://pulsar.princeton.edu/tempo

[13] Taylor J and Weisberg J 1989 Ap. J. 345 p 434-450

[14] Lange Ch et al 2001 Mon. Not. R. Astron. Soc. 326 p 274

[15] Bejger M and Haensel P 2003 A. $\mathscr{E}$ A. 405 p 747-751 